International Journal of Pure and Applied Mathematics

Volume 94 No. 3 2014, 323-339

ISSN: $1311-8080$ (printed version); ISSN: 1314-3395 (on-line version)

url: http://www.ijpam.eu

doi: http://dx.doi.org/10.12732/ijpam.v94i3.3

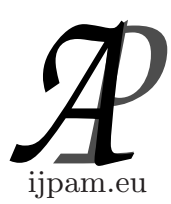

\title{
INTERACTION OF PULSATILE FLOW ON THE PERISTALTIC MOTION OF A MAGNETO-MICROPOLAR FLUID THROUGH POROUS MEDIUM IN A FLEXIBLE CHANNEL: BLOOD FLOW MODEL
}

\author{
Kh.S. Mekheimer ${ }^{1}$, Mohamed S. Mohamed ${ }^{2}$ \\ ${ }^{1,2}$ Mathematics Department \\ Faculty of Science \\ Taif University \\ Hawia, 888 Taif, SAUDI ARABIA \\ ${ }^{2}$ Mathematics Department \\ Faculty of Science \\ Al-Azhar University \\ Nasr City, 11448, Cairo, EGYPT
}

\begin{abstract}
We carry out the effect of the pulsatile flow on peristaltic motion of an incompressible conducting micropolar fluid through porous medium in a channel bounded by flexible walls.Problem formulation in a wave frame of reference is presented.

An oscillatory time dependent flux is being imposed on the peristaltic flow. The non-linear equations governing the flow through porous medium under the effect of a constant magnetic field are solved by using the perturbation method to the second-order in the Womersley number.

Explicit expressions of the pressure gradient, the stream function and the micro-rotation velocity are derived. Numerical calculations are carried out for the pressure rise and friction force on the wall. Also we study the effect of the embedded parameters on the axial fluid velocity. The features of the flow characteristics are analyzed by plotting graphs and discussed in detail. A moti-
\end{abstract}

Received: October 31, 2013

(c) 2014 Academic Publications, Ltd. url: www.acadpubl.eu 
vation of the present analysis is the hope that such a problem will be applicable in many clinical applications.

AMS Subject Classification: $53 \mathrm{C} 11,76 \mathrm{~S} 05$

Key Words: peristaltic flow, pulsatile flow, micropolar fluid, porous medium, MHD

\section{Introduction}

Peristaltic pumping is a mechanism of the fluid transport in a flexible tube by a progressive wave of contraction or expansion from a region of lower pressure to higher pressure. Peristalsis is one of the major mechanisms for fluid in many biological systems. It is an automatic and vital process that moves food through the digestive tract, urine from the kidneys through the ureters into the bladder, and bile from the gallbladder into the duodenum and transport of blood through the artery with mild stenosis. In addition, peristaltic pumping occurs in many practical applications involving biomechanical systems. The peristaltic transport with long wavelength at low Reynolds number [1-3] with all Reynolds number [4] or for long and short wavelength [5] have been analyzed.

Micropolar fluid is a special type of non-Newtonian fluid with microstructure which belongs to a class of fluids with non symmetrical stress sensor referred to as polar fluids. Physically, it represents fluids consisting of randomly oriented particles suspended in a viscous medium. Such a fluid can support couple stresses, body couples and exhibits micro-rotational and micro-inertial effects. It is well known that many physiological fluids behave like suspensions of deformable or rigid particles in a Newtonian fluid. Blood, for instance, is a suspension of red cells, white cells and platelets in plasma and cervical mucus is a suspension of macro-molecules in water like liquid. The theory of micropolar fluid was given by Eringen [6]. Devi and Devanathan [7] investigated peristaltic transport of micro-polar fluids in a cylindrical tube caused by a sinusoidal wave of small amplitude. Philip and Chandra [8] studied peristaltic transport of a simple micro-polar fluid which accounts for micro rotation and micro stretching of the particles contained in a small volume element, using long wavelength approximation. Recently, Mekheimer and Elkot [9] modeled The micropolar fluid for blood flow through a tapered arteries with a stenosis. Mekheimer and Abd elmaboud [10] studied the influences of a micropolar fluid on peristaltic transport in an annulus, as an application of a clot model. Also, the effect of the induced magnetic field was investigated by Mekheimer [11] on the peristaltic flow of a magneto-micropolar fluid. Mekheimer et al. find the similarity Solu- 
tion for flow of a micropolar fluid through a porous medium. Moreover, Hayat and Ali [13] and [14] reported the effects of an endoscope and channel asymmetry on peristaltic transport of micropolar fluids. The non-Newtonian effects of different types of fluids [15-24] or of the Nonlinear and curvature effects [25] on the peristaltic flow have been studied. In most these works, the flow in the wave frame is supposed steady and the associated phenomenon is the transport which results the dynamic interaction between the flow of fluid and the motion of the wall of the channel/tube. But in [26] Srivastava studied the interaction of peristaltic flow with pulsatile flow when the frequency of the travelling wave and that of the imposed pressure gradient are equal. Afifi and Gad [2728 ] studied the interaction of peristaltic flow with pulsatile fluid (respectively Magneto-fluid) through a porous medium when the ratio between these two frequencies is equal to the wave number of the imposed pressure gradient wave. Abd elmaboud and Mekheimer [29] investigated the characteristic for unsteady pulsatile flow through a vertical constricted annulus with heat transfer. Kumar and Prasad [30] studied the interaction of pulsatile flow on the peristaltic motion of couple stress fluid through porous medium. In the present work, we study the effect of the interaction between a pulsatile flow and peristaltic transport of a magneto micropolar fluid in a channel with porous media where we suppose that the flow rate imposed, in this frame, is a function versus time. The analytical solution of this problem is given as an asymptotic expansion in the Womersley number $\beta$ which characterize the unsteadies effect in the wave frame. From this solution we can determine these unsteadies effect on the relation pressure rise - flow rate and on the relation friction force - flow rate often researched for this type of transport.

\section{Analysis}

It is assumed that blood flow is represented by an incompressible micropolar fluid of constant viscosity $\mu$ and density $\rho$. The constitutive equations for the micropolar fluid define the stress tensor $T=\left\{T_{i j}\right\}$ is a nonsymmetric tensor and the couple stress tensor $C=\left\{C_{i j}\right\}$ as

$$
\begin{gathered}
T_{i j}=\left(-p+\lambda \mathbf{V}_{k, k}\right) \delta_{i j}+\mu\left(\mathbf{V}_{i, j}+\mathbf{V}_{j, i}\right)+\kappa\left(\mathbf{V}_{j, i}-\varepsilon_{i j k} \mathbf{w}_{k}\right), \\
C_{i j}=\alpha \mathbf{w}_{k, k} \delta_{i, j}+\beta \mathbf{w}_{i, j}+\gamma \mathbf{w}_{j, i} .
\end{gathered}
$$

Where the symbols denotes: $p$ - pressure, $\lambda, \mu, \kappa$ - coefficients of bulk, shear and vortex viscosities, $\alpha, \beta, \gamma$ - respective coefficients of viscosities, $\varepsilon_{i j k}$ - the Levi-Civita tensor, $\delta_{i j k^{-}}$the Kronecker delta, $\mathbf{V}$ is the velocity vector, $\mathbf{w}$ is 
the microrotation vector and $($,$) denote the covariant derivative. Here, the$ coefficients satisfy the inequalities [7]

$$
\begin{aligned}
& \kappa>0, \quad 3 \lambda+k+2 \mu>0, \quad 2 \mu+k>0, \quad 3 \alpha+2 \gamma>0, \\
& -\gamma<\beta<\gamma, \quad \gamma>0
\end{aligned}
$$

An alternative form to (1) is

$$
\left.T_{i j}=\left(-p+\lambda \mathbf{V}_{k, k}\right) \delta_{i j}+(\mu+\kappa / 2)\left(\mathbf{V}_{i, j}+\mathbf{V}_{j, i}\right)+\kappa\left(\mathbf{V}_{j, i}-\mathbf{V}_{j, i}\right)-\kappa \varepsilon_{i j k} \mathbf{w}_{k}\right),
$$

and the symmetric part of the stress tensor $T$ in (4) is

$$
T_{i j}^{[s]}=\left(-p+\lambda \mathbf{V}_{k, k}\right) \delta_{i j}+(\mu+\kappa / 2)\left(\mathbf{V}_{i, j}+\mathbf{V}_{j, i}\right)
$$

The equations governing the steady flow of an incompressible micropolar fluid in the absence of body force and body couple are

$$
\begin{gathered}
\nabla \cdot \mathbf{V}=0 \\
\rho(\mathbf{V} \cdot \nabla \mathbf{V})=-\nabla p+\kappa \nabla \times \mathbf{w}+(\mu+\kappa) \nabla^{2} \mathbf{V}, \\
\rho j(\mathbf{V} \cdot \nabla \mathbf{w})=-2 \kappa \mathbf{w}+\kappa \nabla \times \mathbf{V}-\gamma(\nabla \times \nabla \times \mathbf{w})+(\alpha+\beta+\gamma) \nabla(\nabla \cdot \mathbf{w})
\end{gathered}
$$

\section{Statement of Physical Model}

We consider peristaltic flow of an incompressible micropolar fluid through a porous channel of width $2 \mathrm{a}$ in the presence of a magnetic field. Let $\mathrm{Y}= \pm \mathrm{h}$ be the upper and lower boundaries of the channel. The motion is considered to be induced by sinusoidal wave trains propagating along the channel walls with a constant speed c, such that

$$
h^{\prime}\left(X^{\prime}, t^{\prime}\right)=a+b \cos \frac{2 \pi}{\lambda}\left(X^{\prime}-c t^{\prime}\right)
$$

where $b, \lambda, c$ are amplitude, wavelength and velocity of the wave and $t$ is time. The flow is unsteady in the laboratory frame $\left(X^{\prime}, Y^{\prime}\right)$ and steady in the wave frame $\left(x^{\prime}, y^{\prime}\right)$ moving with velocity $c$. The relation between laboratory frame and wave frame is given by

$$
x^{\prime}=X^{\prime}-c t^{\prime}, \quad y^{\prime}=Y^{\prime}, \quad u^{\prime}=U^{\prime}-c, \quad v^{\prime}=V^{\prime} .
$$


In the absence of body forces and the body couple, the governing equations (in the wave frame) of an incompressible magneto-micropolar fluid through a porous medium, neglecting the induced magnetic field, are given by:

$$
\begin{aligned}
& \rho\left(\frac{\partial u^{\prime}}{\partial t^{\prime}}+u^{\prime} \frac{\partial u^{\prime}}{\partial x^{\prime}}+v^{\prime} \frac{\partial u^{\prime}}{\partial y^{\prime}}\right)=-\frac{\partial p^{\prime}}{\partial x^{\prime}}+(\mu+k)\left(\frac{\partial^{2} u^{\prime}}{\partial x^{\prime 2}}+\frac{\partial^{2} u^{\prime}}{\partial y^{\prime 2}}\right)+k \frac{\partial w^{\prime}}{\partial y^{\prime}} \\
& -\frac{\mu}{k^{*}}\left(u^{\prime}+c\right)-\sigma B_{0}^{2}\left(u^{\prime}+c\right) \\
& \rho\left(\frac{\partial v^{\prime}}{\partial t^{\prime}}+u^{\prime} \frac{\partial v^{\prime}}{\partial x^{\prime}}+v^{\prime} \frac{\partial v^{\prime}}{\partial y^{\prime}}\right)=-\frac{\partial p^{\prime}}{\partial y^{\prime}}+\mu\left(\frac{\partial^{2} v^{\prime}}{\partial x^{\prime 2}}+\frac{\partial^{2} v^{\prime}}{\partial y^{\prime 2}}\right)-k \frac{\partial w^{\prime}}{\partial x^{\prime}}-\frac{\mu}{k^{*}} v^{\prime} \\
& \rho j\left(\frac{\partial w^{\prime}}{\partial t^{\prime}}+u^{\prime} \frac{\partial w^{\prime}}{\partial x^{\prime}}+v^{\prime} \frac{\partial w^{\prime}}{\partial y^{\prime}}\right)=-k\left(2 w^{\prime}+\frac{\partial u^{\prime}}{\partial y^{\prime}}-\frac{\partial v^{\prime}}{\partial x^{\prime}}\right)+\gamma\left(\frac{\partial^{2} w^{\prime}}{\partial y^{2}}+\frac{\partial^{2} w^{\prime}}{\partial x^{\prime 2}}\right)
\end{aligned}
$$

For further analysis, we use the following non-dimensional variables and parameters:

$$
\begin{aligned}
& x=\frac{x^{\prime}}{\lambda}, y=\frac{y^{\prime}}{a}, u=\frac{u^{\prime}}{c}, v=\frac{\lambda v^{\prime}}{a c}, h=\frac{h^{\prime}}{a}, p=\frac{a^{2} p^{\prime}}{\lambda \mu c}, t=\frac{t^{\prime}}{T_{0}}, \psi=\frac{\psi^{\prime}}{a c}, \\
& w=\frac{a w^{\prime}}{c}, J=\frac{j}{a^{2}}, \phi=\frac{a}{b}, k^{\prime *}=\frac{k^{*}}{a^{2}}, H=\sqrt{\frac{\sigma}{\mu}} a B_{0}, R=\frac{\rho a c}{\mu}, \beta=\frac{\rho a^{2}}{\mu T_{0}} .
\end{aligned}
$$

Using the non-dimensional variables and parameters given above in equation (12), we find that the equations which govern the flow for a magneto micropolar fluid through a porous medium in terms of the stream function $\psi(x, y, t)$ are:

$$
\begin{gathered}
(1-N) \beta \frac{\partial^{2} \psi}{\partial t \partial y}+\delta R(1-N)\left(\frac{\partial \psi}{\partial y} \frac{\partial^{2} \psi}{\partial x \partial y}-\frac{\partial \psi}{\partial x} \frac{\partial^{2} \psi}{\partial y^{2}}\right)=-(1-N) \frac{\partial p}{\partial x} \\
+\delta^{2} \frac{\partial^{3} \psi}{\partial x^{2} \partial y}+\frac{\partial^{3} \psi}{\partial y^{3}}+N \frac{\partial w}{\partial y}-(1-N) \gamma^{2}\left(\frac{\partial \psi}{\partial y}+1\right) \\
-\delta^{2}(1-N) \frac{\partial^{2} \psi}{\partial t \partial x}-\delta^{3} R(1-N)\left(\frac{\partial \psi}{\partial y} \frac{\partial^{2} \psi}{\partial x^{2}}+\frac{\partial \psi}{\partial x} \frac{\partial^{2} \psi}{\partial y \partial x}\right)=-(1-N) \frac{\partial p}{\partial y} \\
+\delta^{4} \frac{\partial^{3} \psi}{\partial x^{3}}+\delta^{2} \frac{\partial^{3} \psi}{\partial y^{2} \partial x}-\delta^{2} N \frac{\partial w}{\partial x}+\frac{(1-N)}{k^{*}} \delta \frac{\partial \psi}{\partial x}, \\
\beta \frac{\partial w}{\partial t}+\delta R\left(\frac{\partial \psi}{\partial y} \frac{\partial w}{\partial x}-\frac{\partial \psi}{\partial x} \frac{\partial w}{\partial y}\right)=-\frac{N}{J(1-N)}\left(2 w+\frac{\partial^{2} \psi}{\partial y^{2}}-\delta^{2} \frac{\partial^{2} \psi}{\partial x^{2}}\right) \\
+\frac{(2-N) N}{J m^{2}(1-N)}\left(\frac{\partial^{2} w}{\partial y^{2}}+\delta^{2} \frac{\partial^{2} w}{\partial x^{2}}\right)
\end{gathered}
$$


where $u=\frac{\partial \psi}{\partial y}, v=-\frac{\partial \psi}{\partial x} N=\frac{k}{k+\mu}$, is the coupling number $0 \leq N \leq 1$ and $m^{2}=\frac{a^{2} k(2 \mu+k)}{\gamma(\mu+k)}$ is the micropolar parameter, $\mathrm{H}$ is the Hartmann number and $k^{*}$ the permeability parameter.

Eliminating the pressure and take $\gamma^{2}=H+\frac{1}{k^{*}}$, we can write:

$$
\begin{aligned}
& (1-N) \beta \nabla^{2} \psi_{t}+\delta R(1-N)\left(\psi_{y} \nabla^{2} \psi_{x}-\psi_{x} \nabla^{2} \psi_{y}\right)=\nabla^{2} \nabla^{2} \psi \\
& +N \nabla^{2} w-(1-N) \gamma^{2} \psi_{y y}, \\
& \beta j\left(\frac{1-N}{N}\right) \frac{\partial w}{\partial t}+\delta R j\left(\frac{1-N}{N}\right)\left(\psi_{y} \frac{\partial}{\partial x}\right. \\
& \left.-\psi_{x} \frac{\partial}{\partial y}\right) w=-2 w-\nabla^{2} \psi+\frac{2-N}{m^{2}} \nabla^{2} w .
\end{aligned}
$$

In the laboratory frame, the dimensional volume flow rate is defined as

$$
Q\left(X^{\prime}, t^{\prime}\right)=\int_{0}^{h^{\prime}} U^{\prime}\left(X^{\prime}, Y^{\prime}, t^{\prime}\right) d Y^{\prime}
$$

where $h^{\prime}=h^{\prime}\left(X^{\prime}, t^{\prime}\right)$, which, in the wave frame, may be expressed as

$$
q\left(x^{\prime}\right)=\int_{0}^{h^{\prime}} u^{\prime}\left(x^{\prime}, y^{\prime}\right) d y^{\prime}
$$

where $h^{\prime}=h^{\prime}\left(x^{\prime}\right)$. Equations (14 and 15) yield

$$
Q\left(X^{\prime}, t^{\prime}\right)=q\left(x^{\prime}\right)+c h^{\prime}\left(X^{\prime}, t^{\prime}\right)
$$

The time- averaged mean flow rate over a period $T$ at a fixed position $X^{\prime}$ is given by

$$
Q\left(X^{\prime}\right)=\frac{1}{T} \int_{0}^{T} Q\left(X^{\prime}, t^{\prime}\right) d t^{\prime}
$$

Using (17) into (18),we get

$$
\begin{gathered}
Q\left(X^{\prime}\right)=q\left(x^{\prime}\right)+a c \\
\theta(t)=\frac{Q\left(X^{\prime}\right)}{a c}, F(t)=\frac{q\left(x^{\prime}\right)}{a c}
\end{gathered}
$$

where $\theta(t)$ and $F(t)$ are respectively the dimensionless flow rates in the fixed and the wave frames. The corresponding dimensionless boundary conditions in the wave frame are

$$
\begin{aligned}
& \psi=0, \quad \frac{\partial^{2} \psi}{\partial y^{2}}=0, w=0 \quad \text { at } y=0 \\
& \psi=F(t), \quad \frac{\partial \psi}{\partial y}=-1, w=0 \quad \text { at } y=h
\end{aligned}
$$


where, $\psi=F(t)$ correspond to the imposed oscillatory flux across the channel and $h(x)=1+\phi \sin (2 \pi x)$.

Under the long wavelength and low Reynolds number consideration Mekheimer [11], Hayat and Ali [13] and Shapiro et al [3],the dimensionless equations of the problem are expressed in the following form:

$$
\begin{aligned}
& (1-N) \beta \psi_{y y t}=\psi_{y y y y}+N w_{y y}-\gamma^{2}(1-N) \psi_{y y}, \\
& \beta j\left(\frac{1-N}{N}\right) \frac{\partial w}{\partial t}=-2 w-\psi_{y y}+\frac{(2-N)}{m^{2}} w_{y y} .
\end{aligned}
$$

and the boundary conditions are the same given by equation (20)

\section{Series Solutions}

Considering the mathematical formulation presented in the previous paragraph, we signal that the considered flow is unsteady. This unsteady phenomenon is due to the interaction of pulsatile flow and peristaltic transport. In this work we also assume that the flow rate $F(t)$ in the wave frame resulting of this interaction is given by

$$
F(t)=F_{0}+\beta F_{1}(t)
$$

where $F_{0}$ is the flow rate in the wave frame in absence of the pulsatile flow and $F_{1}(t)$ is a function of time of frequency. Therefore we seek the solution of the problem under small womersly number $\beta$, we expand $\psi, w$ and $p$ in the following form:

$$
\begin{aligned}
& \psi=\psi_{0}+\beta \psi_{1}+\beta^{2} \psi_{2} \\
& w=w_{0}+\beta w_{1}+\beta^{2} w_{2} \\
& p=p_{0}+\beta p_{1}+\beta^{2} p_{2}
\end{aligned}
$$

Substituting (23) and (22) into (21) and (20) and collecting terms of equal powers of $\beta$,we obtain the following set of the problem.

\section{Zeroth order system}

$$
\begin{gathered}
\frac{\partial^{4} \psi_{0}}{\partial y^{4}}+N \frac{\partial^{2} w_{0}}{\partial y^{2}}-(1-N) \gamma^{2} \frac{\partial^{2} \psi_{0}}{\partial y^{2}}=0 \\
\frac{2-N}{m^{2}} \frac{\partial^{2} w_{0}}{\partial y^{2}}-\left(2 w_{0}+\frac{\partial^{2} \psi_{0}}{\partial y^{2}}\right)=0 \\
\psi_{0}=0 \quad, \quad \psi_{0 y y}=0, \quad w_{0}=0 \quad \text { at } \quad y=0 \\
\psi_{0}=F_{0}, \quad \psi_{0 y}=-1, \quad w_{0}=0 \quad \text { at } y=h
\end{gathered}
$$


First order system

$$
\begin{gathered}
\frac{\partial^{4} \psi_{1}}{\partial y^{4}}+N \frac{\partial^{2} w_{1}}{\partial y^{2}}-(1-N) \gamma^{2} \frac{\partial^{2} \psi_{1}}{\partial y^{2}}=0, \\
\frac{2-N}{m^{2}} \frac{\partial^{2} w_{1}}{\partial y^{2}}-\left(2 w_{1}+\frac{\partial^{2} \psi_{1}}{\partial y^{2}}\right)=0 \\
\psi_{1}=0 \quad, \quad \psi_{1 y y}=0, w_{1}=0 \quad \text { at } y=0 \\
\psi_{1}=F_{1} \quad, \quad \psi_{1 y}=0, w_{1}=0 \quad \text { at } y=h
\end{gathered}
$$

\section{Second order system}

$$
\begin{gathered}
\frac{\partial^{4} \psi_{2}}{\partial y^{4}}+N \frac{\partial^{2} w_{2}}{\partial y^{2}}-(1-N) \gamma^{2} \frac{\partial^{2} \psi_{2}}{\partial y^{2}}=(1-N) \frac{\partial^{3} \psi_{1}}{\partial t \partial y^{2}} \\
\frac{2-N}{m^{2}} \frac{\partial^{2} w_{2}}{\partial y^{2}}-\left(2 w_{2}+\frac{\partial^{2} \psi_{2}}{\partial y^{2}}\right)=\frac{J(1-N)}{N} \frac{\partial w_{1}}{\partial t} \\
\psi_{2}=0 \quad, \quad \psi_{2 y y}=0, w_{2}=0 \quad \text { at } y=0 \\
\psi_{2}=0 \quad, \quad \psi_{2 y}=0, w_{2}=0 \quad \text { at } y=h
\end{gathered}
$$

\section{Solution of the zeroth order system}

From equation (26) by integrating (25) with respect to $y$ and then substituting into (26) and by some calculations the solutions of equations (25 and 26) with the corresponding boundary conditions are:

$$
\begin{aligned}
w_{0}= & \frac{\gamma^{2} m^{2}\left(F_{0}+h\right)(1-N)}{(2-N) Z_{1}}\left(\frac{\sinh \left(\theta_{2} y\right)}{\sinh \left(\theta_{2} h\right)}-\frac{\sinh \left(\theta_{1} y\right)}{\sinh \left(\theta_{1} h\right)}\right) \\
\psi_{0}= & \frac{F_{0}+h}{Z_{1}}\left(\xi_{2}^{2} \frac{\sinh \left(\theta_{2} y\right)}{\sinh \left(\theta_{2} h\right)}-\xi_{1}^{2} \frac{\sinh \left(\theta_{1} y\right)}{\sinh \left(\theta_{1} h\right)}\right) \\
& -y\left(1+\frac{F_{0}+h}{Z_{1}}\left(\xi_{2}^{2} \theta_{2} \operatorname{coth}\left(\theta_{2} h\right)-\xi_{1}^{2} \theta_{1} \operatorname{coth}\left(\theta_{1} h\right)\right)\right)
\end{aligned}
$$

Here

$$
Z_{1}=\xi_{2}^{2}\left(1-\theta_{2} h \operatorname{coth}\left(\theta_{2} h\right)\right)-\xi_{1}^{2}\left(1-\theta_{1} h \operatorname{coth}\left(\theta_{1} h\right)\right)
$$




\section{Solution of the first order system}

The solution of equation(27) with the corresponding boundary conditions are:

$$
\begin{aligned}
w_{1}= & \frac{F_{1} m^{2} \gamma^{2}(1-N)}{Z_{1}(2-N)}\left(\frac{\sinh \left(\theta_{2} y\right)}{\sinh \left(\theta_{2} h\right)}-\frac{\sinh \left(\theta_{1} y\right)}{\sinh \left(\theta_{1} h\right)}\right), \\
\psi_{1}= & \frac{F_{1}}{Z_{1}}\left(\xi_{2}^{2} \frac{\sinh \left(\theta_{2} y\right)}{\sinh \left(\theta_{2} h\right)}-\xi_{1}^{2} \frac{\sinh \left(\theta_{1} y\right)}{\sinh \left(\theta_{1} h\right)}\right) \\
& -y \frac{F_{1}}{Z_{1}}\left(\xi_{2}^{2} \theta_{2} \operatorname{coth}\left(\theta_{2} h\right)-\xi_{1}^{2} \theta_{1} \operatorname{coth}\left(\theta_{1} h\right)\right)
\end{aligned}
$$

\section{Solution of the second order system}

The solutions of equation(29) and with the boundary conditions (30) are:

$$
\begin{aligned}
& w_{2}=A_{31} \sinh \left(\theta_{1} y\right)+A_{32} \sinh \left(\theta_{2} y\right) \\
& +\frac{F_{1}}{Z_{1}}\left(\beta_{1}^{*} \frac{\sinh \left(\theta_{1} y\right)}{\sinh \left(\theta_{1} h\right)}+\beta_{2}^{*} \frac{\sinh \left(\theta_{2} y\right)}{\sinh \left(\theta_{2} h\right)}\right), \\
& \psi_{2}=\frac{(2-N) \dot{F}_{1}}{m^{2} \gamma^{2}(1-N)}\left(\xi_{1}^{2} A_{31} \sinh \left(\theta_{1} y\right)+\xi_{2}^{2} A_{32} \sinh \left(\theta_{2} y\right)\right) \\
& +\frac{F_{1}}{Z_{1}}\left(\lambda_{1} \frac{\sinh \left(\theta_{1} y\right)}{\sinh \left(\theta_{1} h\right)}+\lambda_{2} \frac{\sinh \left(\theta_{2} y\right)}{\sinh \left(\theta_{2} h\right)}\right)+\frac{\dot{F}_{1} y}{Z_{1} \gamma^{2}} Z_{2}-\frac{\dot{F}_{1} A_{21}}{\gamma^{2}(1-N)} y .
\end{aligned}
$$

where

$$
\begin{aligned}
& A_{31}=\frac{\sigma}{Z_{1}^{2} \sinh \left(\theta_{1} h\right)}, \quad A_{32}=\frac{-\sigma}{Z_{1}^{2} \sinh \left(\theta_{2} h\right)}-\frac{\mu}{Z_{1} \sinh \left(\theta_{1} h\right)}, \\
& A_{21}=\frac{2-N}{m^{2}}\left(A_{31} \xi_{1}^{2} \theta_{1} \cosh \left(\theta_{1} h\right)+A_{32} \xi_{2}^{2} \theta_{2} \cosh \left(\theta_{2} h\right)\right) \\
& +\frac{\gamma^{2}(1-N)}{Z_{1}}\left(\lambda_{1} \theta_{1} \operatorname{coth}\left(\theta_{1} h\right)+\lambda_{2} \theta_{2} \operatorname{coth}\left(\theta_{2} h\right)+\frac{Z_{2}}{\gamma^{2}}\right), \\
& \sigma=\xi_{2}^{2} \mu\left(\theta_{2} h \operatorname{coth}\left(\theta_{2} h\right)-1\right) \\
& -\frac{m^{2} \gamma^{2}(1-N)}{(2-N)}\left(h \lambda_{1} \theta_{1} \operatorname{coth}\left(\theta_{1} h\right)+h \lambda_{2} \theta_{2} \operatorname{coth}\left(\theta_{2} h\right)-\lambda_{1}-\lambda_{2}\right), \\
& \mu=\beta_{1}+\beta_{2}, \quad \beta_{1}=\frac{J(1-N)^{3} m^{4} \gamma^{4}}{(2-N)^{2}}-\frac{J(1-N)^{2} m^{4} \gamma^{2} \theta_{1}^{2}}{N(2-N)^{2}}-\frac{(1-N) m^{2} \theta_{1}^{2} \xi_{1}^{2}}{(2-N)}, \\
& \beta_{2}=\frac{-J(1-N)^{3} m^{4} \gamma^{4}}{(2-N)^{2}}+\frac{J(1-N)^{2} m^{4} \gamma^{2} \theta_{2}^{2}}{N(2-N)^{2}}+\frac{(1-N) m^{2} \theta_{2}^{2} \xi_{2}^{2}}{(2-N)}, \\
& \lambda_{1}=\beta_{1} \xi_{1}^{2} \frac{2-N}{m^{2} \gamma^{2}(1-N)}+\frac{\xi_{1}^{2}}{\gamma^{2}}+\frac{J m^{2}(1-N)}{N(2-N)},
\end{aligned}
$$




$$
\begin{aligned}
& \lambda_{2}=\beta_{2} \xi_{2}^{2} \frac{2-N}{m^{2} \gamma^{2}(1-N)}-\frac{\xi_{1}^{2}}{\gamma^{2}}-\frac{J m^{2}(1-N)}{N(2-N)} \\
& \beta_{i}^{*}=\frac{\beta_{i}}{\theta_{i}^{4}-\beta \theta_{i}^{2}+\lambda}, \\
& \beta=\gamma^{2}(1-N)+m^{2}, \quad \lambda=2 \gamma^{2} \frac{(1-N) m^{2}}{(2-N)}, \\
& Z_{2}=\xi_{2}^{2} \theta_{2} \operatorname{coth}\left(\theta_{2} h\right)-\xi_{1}^{2} \theta_{1} \operatorname{coth}\left(\theta_{1} h\right), \\
& \theta_{i}=\frac{1}{\sqrt{2}} \sqrt{\beta_{r} \pm \sqrt{\beta_{r}^{2}-4 \lambda}}, \quad \xi_{i}^{2}=\theta_{i}^{2}-m^{2}, \\
& \beta_{r}=(1-N) \gamma^{2}+m^{2}, \quad \lambda=\frac{2 m^{2}(1-N) \gamma^{2}}{(2-N)}, \quad i=1,2 .
\end{aligned}
$$

Thus the stream function and the microrotation velocity component $\mathrm{w}$ will take the forms

$$
\begin{aligned}
w(x, y, t)= & \frac{\gamma^{2} m^{2}(F(t)+h)(1-N)}{(2-N) Z_{1}}\left(\frac{\sinh \left(\theta_{2} y\right)}{\sinh \left(\theta_{2} h\right)}-\frac{\sinh \left(\theta_{1} y\right)}{\sinh \left(\theta_{1} h\right)}\right) \\
& +\beta F_{1}\left(A_{31} \sinh \left(\theta_{1} y\right)+A_{32} \sinh \left(\theta_{2} y\right)\right. \\
& +\frac{1}{Z_{1}}\left(\beta_{1}^{*} \frac{\sinh \left(\theta_{1} y\right)}{\sinh \left(\theta_{1} h\right)}+\beta_{2}^{*} \frac{\sinh \left(\theta_{2} y\right)}{\left.\sinh \left(\theta_{2} h\right)\right)}\right. \\
\psi(x, y, t)= & \frac{F(t)+h}{Z_{1}}\left(\xi_{2}^{2} \frac{\sinh \left(\theta_{2} y\right)}{\sinh \left(\theta_{2} h\right)}-\xi_{1}^{2} \frac{\sinh \left(\theta_{1} y\right)}{\sinh \left(\theta_{1} h\right)}\right) \\
& -y\left(1+\frac{F(t)+h}{Z_{1}}\left(\xi_{2}^{2} \theta_{2} \operatorname{coth}\left(\theta_{2} h\right)-\xi_{1}^{2} \theta_{1} \operatorname{coth}\left(\theta_{1} h\right)\right)\right) \\
& +\frac{(2-N) \dot{F}_{1}}{m^{2} \gamma^{2}(1-N)}\left(\xi_{1}^{2} A_{31} \sinh \left(\theta_{1} y\right)+\xi_{2}^{2} A_{32} \sinh \left(\theta_{2} y\right)\right) \\
& +\frac{\dot{F}_{1}}{Z_{1}}\left(\lambda_{1} \frac{\sinh \left(\theta_{1} y\right)}{\sinh \left(\theta_{1} h\right)}+\lambda_{2} \frac{\sinh \left(\theta_{2} y\right)}{\sinh \left(\theta_{2} h\right)}\right) \\
& +\frac{F_{1} y}{Z_{1} \gamma^{2}} Z_{2}-\frac{F_{1} A_{21}}{\gamma^{2}(1-N)} y .
\end{aligned}
$$

\section{Pressure Rise and Shear Stress}

When the flow is steady in the wave frame, one can characterize the pumping performance by means of the the pressure rise per wavelength. So, the axial 
pressure gradient can be obtained from the equation

$$
\frac{\partial p}{\partial x}=\frac{1}{(1-N)}\left(\frac{\partial^{3} \psi}{\partial y^{3}}+N \frac{\partial w}{\partial y}\right)-\beta \frac{\partial^{2} \psi}{\partial t \partial y}-\gamma^{2}\left(\frac{\partial \psi}{\partial y}+1\right)
$$

So, the axial pressure gradient will take the form

$$
\begin{aligned}
\frac{\partial p}{\partial x}= & \frac{F(t)+h}{Z_{1}}\left(\Omega_{2} \frac{\cosh \left(\theta_{2} y\right)}{\sinh \left(\theta_{2} h\right)}-\Omega_{1} \frac{\cosh \left(\theta_{1} y\right)}{\sinh \left(\theta_{1} h\right)}\right) \\
& -\gamma^{2}\left(\frac{F(t)+h}{Z_{1}}\right)\left(\xi_{2}^{2} \theta_{2} \operatorname{coth}\left(\theta_{2} h\right)-\xi_{1}^{2} \theta_{1} \operatorname{coth}\left(\theta_{1} h\right)\right) \\
& +\beta \frac{(2-N) \dot{F}_{1}}{m^{2} \gamma^{2}(1-N)}\left(\xi_{1}^{2} \theta_{1}^{3} A_{31} \cosh \left(\theta_{1} y\right)+\xi_{2}^{2} A_{32} \theta_{2}^{3} \cosh \left(\theta_{2} y\right)\right) \\
& +\beta \frac{\dot{F}_{1}}{Z_{1}(1-N)}\left(\lambda_{1} \theta_{1}^{3} \operatorname{coth}\left(\theta_{1} h\right)\right. \\
& \left.+\lambda_{2} \theta_{2}^{3} \operatorname{coth}\left(\theta_{2} h\right)\right)+\beta \frac{F_{1} N}{(1-N)}\left(\theta_{1} A_{31} \cosh \left(\theta_{1} y\right)+\theta_{2} A_{32} \cosh \left(\theta_{2} y\right)\right. \\
& \left.+\frac{1}{Z_{1}}\left(\beta_{1}^{*} \theta_{1} \operatorname{coth}\left(\theta_{1} h\right)+\beta_{2}^{*} \theta_{2} \operatorname{coth}\left(\theta_{2} h\right)\right)\right) \\
& -\beta \frac{(2-N) \dot{F}_{1}}{m^{2}(1-N)}\left(\xi_{1}^{2} \theta_{1} A_{31} \cosh \left(\theta_{1} y\right)+\xi_{2}^{2} A_{32} \theta_{2} \cosh \left(\theta_{2} y\right)\right) \\
& -\beta \frac{F_{1} \gamma^{2}}{Z_{1}}\left(\lambda_{1} \theta_{1} \operatorname{coth}\left(\theta_{1} h\right)+\lambda_{2} \theta_{2} \operatorname{coth}\left(\theta_{2} h\right)\right) \\
& -\beta \frac{F_{1}}{Z_{1}} Z_{2}+\beta \frac{F_{1}}{(1-N)} A_{21} .
\end{aligned}
$$

Here

$$
\Omega_{i}=\theta_{i}\left(\xi_{i}^{2}\left(\frac{\theta_{i}^{2}}{1-N}-\gamma^{2}\right)+\frac{N \gamma^{2} m^{2}}{2-N}\right), i=1,2
$$

The pressure rise $\Delta p_{\lambda}$ and the friction force $F_{\lambda}$ for a channel of length L in its nondimensional forms are given by

$$
\begin{aligned}
& \Delta p_{\lambda}=\int_{0}^{1}\left(\frac{\partial p}{\partial x}\right) d x \\
& F_{\lambda}=-\int_{0}^{1}\left(\frac{\partial p}{\partial x}\right) d x .
\end{aligned}
$$

The integrals in (38) is not integrable in closed form, it is evaluated numerically using a digital computer. An interesting property of the micropolar fluid 
is that the stress tensor is not symmetric. The nondimensional shear stresses in the problem under consideration are given by

$$
\begin{array}{r}
\tau_{x y}=\frac{\partial^{2} \psi}{\partial y^{2}}-\frac{N}{1-N} w, \\
\tau_{y x}=\left(\frac{1}{1-N}\right) \frac{\partial^{2} \psi}{\partial y^{2}}+\frac{N}{1-N} w
\end{array}
$$

The shear stresses $\tau_{x y}$ and $\tau_{y x}$ are calculated at both the lower and upper walls and their graphical results are shown in figures(8-10).

\section{Numerical Results and Discussion}

We are interested in this work to investigate the effects of $\beta, \varphi, \mathrm{m}, \mathrm{N}, \mathrm{H}, k^{*}$ and time $\mathrm{t}$ with the pulsatile flow, for this the flow rate is given by $\theta(t)=$ $\theta_{0}+\beta \sin (2 \pi t)$.

This section is divided into three subsections. In the first subsection, the effects of various parameters on the pulsating pumping interaction characteristics of a magneto-miropolar fluid through porous medium are investigated. The shear stress characteristics are discussed in the second subsection. The fluid velocity and the micro-rotation profile are illustrated in the last subsection.

\subsection{Pulsating-Pumping Interaction Characteristics}

This subsection describes the influences of various emerging parameters of our analysis on the pressure rise per wavelength $\Delta p_{\lambda}$ and the friction force $F_{\lambda}$ on the wall channel. The effect of these parameters are shown in figures (2-7). These figures describes the variation of $\Delta p_{\lambda}$ and $F_{\lambda}$ with $\theta(t)$ for various values of $\beta, \varphi, \mathrm{m}, \mathrm{N}, \mathrm{H}$ and $k^{*}$. On the first, in the absence of the pulsatile flow the distribution of $\Delta p_{\lambda}$ and $F_{\lambda}$ versus the dimensionless flow rate $\theta$ are illustrated in figures $(2 \mathrm{a}, \mathrm{b})$, we see from these figures that the relation between $\Delta p_{\lambda}, F_{\lambda}$ and $\theta$ are linear and coincide with those found by several authors[1-3,10,11] The graph in figure (2a) is sectored so that the upper right-hand quadrant $I$ denotes the region of peristaltic pumping, where $\theta>0$ (positive pumping) and $\Delta p_{\lambda}>0$ (adverse pressure gradient). Quadrant $I I$, where $\Delta p_{\lambda}<0$ (favorable pressure gradient) and $\theta>0$ (positive pumping), is designated as augmented flow (copumping region). Quadrant $I V$, such that $\Delta p_{\lambda}>0$ (adverse pressure gradient) and $\theta<0$, is called retrograde or backward pumping. The flow is opposite to the direction of the peristaltic motion, and there is no flows in the 
last quadrant (Quadrant $I I I$ ). For the case where the peristaltic transport is superposed to pulsatile flow $(\beta \neq 0)$ and for every positive median value of $\theta_{0}$, abscissa of one point of a straight line $\mathrm{D}$ of the figure (2a) for which $\Delta p_{\lambda}>0$, we observe that the distributions of $\Delta p_{\lambda}$ and $F_{\lambda}$ versus the flow rate $\theta$ become ellipsis whose the principal and the median shafts increase with increasing $\beta$, figure (3a) and where the principal shaft direction changes with increasing $\varphi$, figure (4a). This change in the principal shaft direction is obvious as $\mathrm{N}$ increases, while slightly changes as $\mathrm{m}, \mathrm{H}$ and and $k^{*}$ are increases, figures(5-7). Moreover, from these figures we notice that the pressure rise $\Delta p_{\lambda}$ increases with increasing $\mathrm{N}$ and $\mathrm{H}$, while it decreases as $\mathrm{m}$ and $k^{*}$ increases. These observations constitute already a control aid of the pulsatile flow existence. In addition they translate clearly the influence of pulsatile flow and of the geometric properties of the wall on the peristaltic pumping region. Finally, the dimensionless friction force $F_{\lambda}$ are plotted in figures $(2-6 \mathrm{~b})$, these show that the friction forces have the opposite behaviour compared to pressure rise.

\subsection{The Shear Stress Characteristics}

It is known that the stress tensor is not symmetric in micropolar fluid, that is why the expressions for $\tau_{x y}$ and $\tau_{y x}$ are different. In Figures (8-10), we have plotted the shear stresses $\tau_{x y}$ and $\tau_{y x}$ at the upper and lower walls for various values of the $\beta, \varphi, \mathrm{m}, \mathrm{N}, \mathrm{H}$ and $K^{*}$ for a pulsating $\beta \neq 0$ and non-pulsating $\beta=0$ flow. It can be seen that both shear stress at the channel walls are symmetric about the line $\mathrm{x}=0$. Figure (9) is prepared just to see the effects of $\mathrm{m}$ and $\mathrm{N}$ on the shear stress $\tau_{y x}$ at the lower wall. It is found that shear stress $\tau_{y x}$ decreases with an increase in $\mathrm{m}$ while it increases with an increase in $\mathrm{N}$. The direction of the shear stress is along the direction of the lower wave velocity.

Moreover, the magnitude of the shear stress is greater for a pulsating flow than that for a non-pulsating flow. Also, the shear stress magnitude decreases as $K^{*}$ increases, while it increases as $\mathrm{H}$ increases. Moreover, both shear stresses have directions opposite to the upper wave velocity, while the directions of these shear stresses are along the direction of the lower wave velocity. The shear stress take a linear behavior for $\varphi=0$, solid walls and an oscillatory behavior for a wavy sinusoidal walls, peristaltic pump, figure (8a). 


\subsection{The Fluid and Micro-Rotation Velocity Profile}

Figures (11-12) describe the distribution of the axial fluid velocity $\mathrm{u}(\mathrm{y})$ and the microrotation velocity within y for different values of $\mathrm{H}, K^{*}, \mathrm{~m}$ and $\mathrm{N}$ for a pulsating and non-pulsating flow (steady flow). The graphical results of these figures indicate that the axial velocity profile decreases as $\mathrm{H}$ and $\mathrm{m}$ increase in the region near the center of the channel while it increases for the same values of $\mathrm{H}$ and $\mathrm{m}$ in the region near to the lower and upper walls. However, an opposite behavior is noticed as $\mathrm{N}$ and $K^{*}$ increases. Also, the fluid velocity is increases as the pulsating parameter $\beta$ increases. So, the fluid velocity for a micropolar fluid is higher than that for a Newtonian fluid and the fluid velocity is greater for a pulsating flow than that for a steady one. figure (12 b), indicate that the magnitude of the micro-rotation velocity increases as $\beta$ increases. Further in the half region, the micro-rotation velocity is one direction, and in the other half, it is in the opposite direction and it is zero at $\mathrm{y}=0$.

\section{Acknowledgments}

This paper was funded by the Deanship of Scientific Research (DSR), Taif University (TU), under grant no. (1-434-2325). The authors, therefore, acknowledge technical and financial support of Taif University. The support is in the form of project for academic research at TU.

\section{References}

[1] M. Y. Jaffrin and A. H. Shapiro, Peristaltic pumping. Ann. Rev. Fluid Dyn, 3 (1971), 13 - 37.

[2] M. J. Manton, Long-wavelength peristaltic pumping at lowReynolds number J. Fluid Mech, 68 (1975), 467 - 476.

[3] A. H. Shapiro, M. Y. Jaffrin and S. L.Weinberg, Peristaltic pumping with long wavelengths at low Reynolds number, J. fluid Mech, 37 (1969), 799825 .

[4] T. Colgan and M. R. Terrill, On peristaltic transport of fluids, J. of Theoretical and Applied Mechanics, 6(1) (1987), 3 - 22.

[5] C. Barton and S. Raynor, Peristaltic Flow in Tubes, Bull. Math. Biophys, 30 (1968), 663 - 680. 
[6] A. C. Eringen, Theory of micropolar fluids. J Math Mech, 16 (1966),1-18.

[7] R. Girija Devi and R. Devanathan, Peristaltic transport ofmicropolar fluid, Proceedings of the National Academy of Sciences, India. Section A, 81 (1975), 149163.

[8] D. Philip and P. Chandra, Peristaltic transport of a simple micro fluid, Proc Nat Acad Sci India, 65 (1995),63-74.

[9] Kh. S. Mekheimer and M. A. Elkot, The micropolar fluid model for blood flow through a tapered arteries with a stenosis. Acta Mech. Sin., 24(2008), 637644 .

[10] Kh. S. Mekheimer, and Y. Abd elmaboud, The influences of a micropolar fluid on peristaltic transport in an annulus: Application of a clot model, J. Applied Bionic and Biomech., 5, No 1, (2008), 13-23.

[11] Kh. S. Mekheimer, Peristaltic flow of a magneto-micropolar fluid : Effect of induced magnetic field, J Appllied Mathematics, Article ID 570825, 23 pages (2008)

[12] Kh. S. Mekheimer, S. Z.-A. Husseny, A. T. Ali and R. E. Abo-Elkhair, Similarity Solution for Flow of a Micropolar Fluid Through a Porous Medium, Applications and Applied Mathematics: An International Journal (AAM), Vol. 6, Issue 11 (June 2011) pp. 2082- 2093

[13] Hayat T, Ali N. Effects of an endoscope on peristaltic flow of a micropolar fluid. Math Comput Model, 48 (2008),721-733.

[14] Ali N, Hayat T. Peristaltic flow of a micropolar fluid in an asymmetric channel. Comput Math Appl, 55 (2008), 589-608.

[15] D. Tsiklauri and I. Beresnev. Non-Newtonian effects in the peristaltic flow of a Maxwell fluid, Physical Review E,, 64 (2001), 036303.

[16] T. Hayat, Y. Wang, A. M. Siddiqui and K. Hutter, Peristaltic motion of a Johnson-Segalman fluid in a planar channel, Mathematical Problems in Enginneering, 1 (2003), 1 - 23.

[17] T. Hayat, Y. Wang, K. Hutter, S. Asghar and A. M. Siddiqui, Peristaltic transport of an Oldroyd-B fluid in a planar channel, Mathematical problems in engineering, 4 (2004), 347 - 376. 
[18] T. Hayat, F. M. Mohamed and S. Asghar, Peristaltic flow of a Magnetohydrodynamic Johnson-Segalman fluid, Nonlinear Dynamics, 40 (2005), 375 - 385 .

[19] T. Hayat and N. Ali, Peristaltically induced motion of a MHD third grade fluid in a deformable tube, J. Phys. A, 370 (2006), 225 - 239.

[20] Abd El Hakeem Abd El Naby, A.E.M. El Misery and M.F. Abd El Kareem, Effects of a magnetic field on trapping through peristaltic motion for generalized Newtonian fluid in channel, J. Phys. A,367 (2006), 79 - 92.

[21] Hayat, T, Yasir Khan, Kh S Mekheimer and Nasir Ali, Effect of an induced magnetic field on the Peristaltic flow of a third-order fluid, Numerical Methods of Partial Differential Equations Journal (NMPDE), 26 (2010), 345-366.

[22] Y. Abd elmaboud and Mekheimer Kh. S., Non-linear peristaltic transport of a second-order fluid through a porous medium, Applied Mathematical Modelling, 35 (2011), 2695-2710.

[23] Kh. S Mekheimer, Najma Saleem and T. Hayat, Simultaneous effects of induced magnetic field and heat and mass transfer on the peristaltic motion of second-order fluid in a channel, Accepted for publication , in International Journal for Numerical Methods in Fluids: 70 (2012), 342-358.

[24] Kh. S. Mekheimer, S. R. Komyc and S. I. Abdelsalamd, Simultaneous effects of magnetic field and space porosity on compressible Maxwell fluid transport induced by a surface acoustic wave in a micro-channel , Chin. Phys. B, Vol. 22, No. 12 (2013), http://dx.doi.org/10.1088/16741056/22/12/12XXXX

[25] A. Ramachandra Rao and M. Mishra, Nonlinear and curvature effects on peristaltic flow of a viscous fluid in an asymmetric channel, Acta Mechanica, 168 (2004), $35-59$.

[26] L. M. Srivastava. Interaction of peristaltic flow with pulsatile flow in a circular cylindrical tube. Biomechanics,, 18(4) (1985), 247 - 253.

[27] N. A. S. Afifi and N. S. Gad, Interaction of peristaltic flow with pulsatile fluid through a porous medium, Applied Mathematics and Computation, 142 (2003), 167 - 176. 
[28] N. A. S. Afifi and N. S. Gad, Interaction of peristaltic flow with pulsatile magneto-fluid through a porous medium, Acta Mechanica, 149(2001), 229 $-237$.

[29] Y. Abd elmaboud and Kh. S. Mekheimer, Unsteady pulsatile flow through a vertical Constricted annulus with heat transfer" , Naturforschung A, Z.Naturforsch. 67 (2012), 185-194 /DOI: 10.5560/ZNA.2012-0011I.

[30] S.Ravi kumar and R.Siva Prasad, Interaction of pulsatile flow on the peristaltic motion of couple stress fluid through porous medium in a flexible channel, Eur. J. Pure Appl. Math, 3 (2010), 213-226. 
